\section{Cahiers de Narratologie}

Analyse et théorie narratives

13 | 2006

Nouvelles approches de l'intertextualité

\title{
Choses vues, choses lues : le reportage à l'épreuve de l'intertexte
}

\section{Myriam Boucharenc}

\section{(2) OpenEdition}

1 Journals

Édition électronique

URL : http://journals.openedition.org/narratologie/320

DOI : 10.4000/narratologie.320

ISSN : 1765-307X

Éditeur

LIRCES

\section{Référence électronique}

Myriam Boucharenc, "Choses vues, choses lues : le reportage à l'épreuve de l'intertexte », Cahiers de Narratologie [En ligne], 13 | 2006, mis en ligne le 01 septembre 2006, consulté le 14 novembre 2019. URL : http://journals.openedition.org/narratologie/320; DOI : 10.4000/narratologie.320

Ce document a été généré automatiquement le 14 novembre 2019.

\section{(c) (i) (9)}

Cahiers de Narratologie - Analyse et théorie narratives est mis à disposition selon les termes de la licence Creative Commons Attribution - Pas d'Utilisation Commerciale - Pas de Modification 4.0 International. 


\title{
Choses vues, choses lues : le reportage à l'épreuve de l'intertexte
}

\author{
Myriam Boucharenc
}

1 Au lendemain de la Grande Guerre, sous le choc de l'Histoire, le besoin se fait sentir $d^{\prime}$ '« une nouvelle alliance entre la littérature et le vécu »1. La "crise du concept de littérature » identifiée dès 1924 par Jacques Rivière ${ }^{2}$, se traduit notamment par une remise en question de la fiction - au profit du document et du témoignage - dont le grand reportage a été l'un des plus puissants vecteurs. Ce genre héritier du journalisme d'investigation anglo-saxon, se développe en France depuis la fin du XIX ${ }^{\mathrm{e}}$ siècle, non sans aménagements ni sans concessions, notamment à la tradition plus spécifiquement française de l'Ecriture. Il faudra attendre les Années folles, avec l'évolution des techniques de communication et l'essor de la presse à grand tirage, pour que triomphe «l'envoyé spécial » en " professionnel de l'aventure " ${ }^{3}$ parcourant le monde à la vitesse de l'événement afin de livrer au lecteur du journal le récit tout chaud de son enquête. Journalistes tentés par la littérature et littérateurs en mal d'action font alors succès commun: tandis que les nouveaux journalistes sont souvent des transfuges de la littérature (Albert Londres fut d'abord poète) ou des doubles plumes (Henri Béraud ou les frères Tharaud), nombreux sont les écrivains, parfois même d'avant-garde qui, plus ou moins durablement, se tournent vers le reportage : Dorgelès, Mac Orlan, Carco mais aussi Cendrars, Soupault ou Vailland... L'interpénétration des milieux littéraires et journalistiques, qu'incarne au plus haut point Kessel, auteur de L'Equipage, fondateur de l'hebdomadaire Gringoire et reporter à succès des plus grands quotidiens du temps (Le Matin, Paris-Soir) s'intensifie dans l'entre-deux-guerres, portée par la vogue éditoriale des collections de reportages qui, chez Albin Michel ou aux Editions de France, assurent la promotion de l'écrit journalistique au statut, plus littéraire et plus durable, du livre. Ainsi la «grandeur » du "grand reportage » tient-elle, autant qu'à sa vocation des lointains, à l'intérêt que les écrivains lui portent.

2 Le reportage semble ainsi pris en otage entre un journalisme qui compte sur lui pour asseoir la profession et une littérature qui y cherche une solution à la littérature. Ce double lien n'est évidemment pas sans incidence sur la configuration d'un «genre » 
empirique qui, faute d'art poétique bien arrêté, se cherche à la fois contre la littérature, dont il entend se distinguer, et par la littérature, à laquelle il prétend faire concurrence. Une ambivalence dont rend compte Henri Béraud, prix Goncourt 1922 et journaliste vedette du Petit Parisien et du Journal, lorsqu'il considère que les « journalistes ne sont pas de "vrais écrivains" ", tout en affirmant que «le reportage sera la littérature de demain $»^{4}$. Une " littérature active », selon l'expression forgée par Mac Orlan, de nature à supplanter aussi bien la tradition du récit de "voyage égoïste $»^{5}$, que celle du roman comme fabrique d'illusion réaliste. En finir avec les filtres de la fiction comme de la médiation culturelle, telle est l'ambition du reportage qui se targue de "coller » aux faits, sans pour autant renoncer aux effets plus littéraires d'une écriture qui, tout en s'adressant à un lectorat élargi, se doit d'exploiter les qualités d'« auteur » sur la célébrité desquelles mise le journal pour lancer l'enquête et en obtenir le succès commercial. Cette double postulation, l'une vers la littéralité (la soumission à l'événement, à la « chose vue »), l'autre vers la littérarité (la soumission de l'événement, la chose "écrite »), pose, de façon cruciale, la question de l'intertextualité, arme en l'occurrence à double tranchant puisqu'elle menace la «chose vue » de se transformer en " chose lue », tout en concourant à l'élaboration du genre et à son inscription dans la tradition des Auteurs.

3 Aussi le reportage entretient-il un rapport hésitant et souvent duplice avec le jeu intertextuel qui, variablement assumé, assure plusieurs fonctions dans la constitution du genre : une fonction polémique, tout d'abord, liée à un souci de démarcation (tracer la frontière entre journalisme et littérature, à une époque où le statut professionnel du journaliste s'impose), une fonction poétique ensuite, lorsque s'exerçant dans le paradigme restreint du genre, l'intertextualité contribue à l'élaboration d'une spécificité générique (fonder un genre nouveau avec ses rituels d'écriture), une fonction génétique enfin, au sens où l'œuvre de l'écrivain bien souvent s'amorce, se poursuit ou simplement se réfléchit dans l'écriture du reportage.

Le reportage contre la littérature

4 Le reportage se situe principalement dans le sillage de deux genres avec lesquels il entretient une relation à la fois conflictuelle et élective : le récit de voyage et le roman d'aventures.

5 Du récit de voyage, le récit de reportage au long court se rapproche parfois jusqu'à s'y méprendre. Et, sauf à recourir à des critères externes, comme la commande du journal, il est souvent malaisé de classer tel récit dans l'un ou l'autre genre tant il est vrai que ce nouveau journalisme emprunte massivement aux techniques narratives du récit de voyage : le récit linéaire mimétique de l'itinéraire ou la forme du journal, la mise en scène du reporter en "coureur des continents ${ }^{6}$, la narration par témoignages interposés et, bien-sûr, les destinations communes aux deux genres. S'attachant à dégager le paradigme du "retour d'URSS ", si caractéristique de l'après-guerre, François Hourmant traite sans distinction du reportage et du récit de voyage ${ }^{7}$. C'est pourtant en s'opposant à ce dernier que le reportage cherche, pour une large part, à se poser.

6 Henri Béraud se fait le porte-parole de la profession lorsqu'il distingue le «flâneur salarié » de "l'écrivain en promenade ou en croisière, auteur de ces relations de voyages que l'on écrit à loisir et que l'on imprime pour la postérité ${ }^{8}$. Le reportage est un voyage finalisé par les besoins de l'information : l'itinéraire et son rythme ne sont pas choisis par le voyageur mais dictés par les nécessités de l'enquête et soumis aux 
exigences de l'actualité. Reprenant à son compte le pacte référentiel caractéristique du récit de voyage, le reportage l'exalte au détriment de l'exotisme littéraire dont il dénonce volontiers l'écueil, à l'instar d'un Morand fustigeant « le lyrisme de la patache ou de la balancelle, la rêvasserie sur les gondoles et la rhétorique du kilomètre " ${ }^{9}$. Les frères Tharaud, pourtant si friands de grands reportages, ne sont, aux yeux d'Armand Paviel, que des voyageurs littéraires qui «parcourent le Maroc ou l'Albanie avec leur bibliothèque portative $»^{10}$. Parce qu'il combine l'évocation des lieux et la mémoire des livres, le récit de voyage manque la vérité du monde. Du moins est-ce ainsi que dans $L a$ Caravane sans chameaux, au titre discrètement polémique, Dorgelès met en scène le contre-modèle du voyageur à l'ombre des livres :

- Curieux spectacle, n'est-ce pas?

- Oh ! oui, s'exclama la plus maigre. Une vraie scène des Mille et une Nuits...

Je faillis lui rire au nez. [...] Encore une qui ne verrait jamais rien qu'à travers ses

lectures et repartirait convaincue que les Orientaux sont tous derviches, chameliers ou muezzins ${ }^{11}$.

7 Le dévoilement progressif de la vanité des illusions et des allusions littéraires contribue à l'élaboration d'un discours du « regard neuf» dont Dorgelès formule l'exigence en guise d'incipit: «Ce n'est pas Hérodote qui a découvert l'Egypte, ni Diodore, ni Marc Aurèle, ni Strabon. C'est moi !». Le reportage entend donc " délittérariser » le récit de voyage pour mieux réaffirmer le primat du référent. Cette surenchère référentielle doublée d'un interdit intertextuel caractérise, pour une part, la "scénographie ${ }^{12}$ propre à ce type de récit.

8 Pour une part, seulement, dans la mesure ou la finalité informative, s'accompagne d'une visée divertissante qui reconduit le reportage vers un mode de saisie littéraire du monde. D'un côté le reporter multiplie les garanties d'authenticité, de l'autre il fait en sorte que le récit de la vérité "tienne le lecteur en haleine ${ }^{13}$, autrement dit que l'enquête se lise "comme un roman » : pour mieux renforcer cet horizon d'attente, la presse accole régulièrement à "reportage » l'adjectif «sensationnel ». Communiquer un témoignage qui procure les plaisirs du roman, suppose que le reportage compose avec la fiction pour devenir ce que Kessel nomme un «roman d'aventure réel $»^{14}$. La dette au romanesque est telle qu'elle finit souvent par éclipser le témoignage : la construction en épisodes, que le journal se charge d'interrompre au moment le plus propice à l'entretien du suspense, les personnes stylisées en personnages et jusqu'à la figure du reporter justicier-enquêteur dont les origines sont à chercher dans la lignée des Alcide Jolivet, Harry Blount et Rouletabille, attestent la fragilité des frontières génériques. A ceci près que "l'effet de réel» qui, dans le roman, fait le jeu de la vraisemblance, risque, dans le reportage, de se transformer en "effet romanesque ", soulignant l'emprunt au lieu d'avérer les faits.

9 La contamination du reportage par le modèle romanesque soulève au moins deux questions. Tout d'abord: comment combiner le modèle référentiel du récit de voyage, tenté par le degré zéro de la dispositio (garant d'une homologie de structure entre le périple et sa narration) et le modèle romanesque caractérisé par la liberté d'invention structurelle? D'autre part, la promiscuité avec le roman, si elle contribue à n'en pas douter au succès du reportage, risque en même temps d'absorber sa spécificité en mettant en péril le pacte référentiel dont il se réclame. Sous peine de tomber de Charybde en Sylla, le reportage doit donc également se démarquer du modèle romanesque. Mais comment? 

la «chose vue »? En déplaçant la fiction, du discours romanesque vers la réalité, en l'assimilant à l'ordre du vécu. Le reportage se plaît à afficher le paradoxe de «l'incroyable mais vrai »:

Tout ce que l'on va trouver rapporté ici est strictement vrai. Il peut paraître étrange que l'auteur d'un reportage éprouve, avant d'entreprendre sa relation, le besoin d'en affirmer l'exactitude. Mais comment pourrai-je faire autrement, lorsque moimême, au souvenir des lieux que j'ai traversés, des hommes que j'ai côtoyés, des lois qui les régissent, je doute de mes sens et de ma raison ? ${ }^{15}$

11 A propos de l'affaire Landru, André Salmon se souvient d'avoir « empli les colonnes du Matin d'un reportage macabre qui eût pu [lui] fournir les éléments d'un conte fantastique $»^{16}$. Jamais, constate Jean Rodes en 1924, «la fiction ne parut à ce point inférieure à ce qui a été réellement vécu ${ }^{17}$. C'est en exploitant toutes les potentialités fictionnelles du réel, que le reporter croit (ou feint de) résoudre l'aporie de l'emprunt aux modalités de la fiction. Une stratégie qui lui permet, en tous cas, de se distinguer à la fois du roman, dont il inverse le principe (non pas donner une fiction du monde, mais prélever la fiction directement dans le réel) et du récit de voyage qui, se heurtant régulièrement à "l'insuffisance des mots ${ }^{18}$, postule une certaine impuissance de l'écriture face au réel, là où le reporter, fort de la politique du " jamais vu, jamais dit ", prétend plonger au cœur du monde pour y trouver les mots. Ainsi se profile l'idée selon laquelle l'écriture du réel serait moins de l'ordre, indirect, de la représentation, que de l'ordre immédiat de la transcription.

12 Au passé de la littérature, comme aux mirages de la fiction, le reportage oppose le présent d'un référent dont il décuple la présence par l'exercice d'un regard. Mû par une quête de l'essence, de la loi ou du type, le récit de voyage unifie volontiers le divers, au risque de ramener l'autre au même et de gommer l'épaisseur temporelle du monde ${ }^{19}$, là où le reportage affiche sa préférence pour la vision en profondeur ( «le profond aujourd'hui » cher à Cendrars) ou le "panorama ». C'est en multipliant ses angles de saisie que, distanciant l'héritage culturel, le reportage invente sa perspective et cerne sa spécificité. Dans son enquête sur l'Unterwelt berlinois, Kessel opte pour la vision burlesque du monde vu à l'envers, accréditant ainsi la sensibilité du reportage à l'accidentel et au refoulé social : bagne, bas-fonds, «prisons de femmes » et « hommes en cages $»^{20}$, « coulisses de la police $»^{21}$ et maisons de fous sont les lieux de prédilections du reporter apprenti-sémiologue et amateur de réalités en marge. A l'imaginaire linéaire du récit de voyage se substitue une représentation démultipliée de l'espace, appelant une véritable traversée de la réalité. «La poésie du "milieu" est morte et [...] dans ce domaine tout romantisme littéraire est périmé $»^{22}$ : Cendrars place le Panorama de la pègre, dans une perspective qui déplace la profondeur du réel, de l'axe diachronique (dont procède l'intertextualité) vers l'axe synchronique (qui valorise les plans simultanés, chers à l'esthétique cubiste). Sous le titre, "Trois hommes en Allemagne", Paris-soir fait paraitre en 1932 une enquête à triple point de vue: Sauerwein rend compte de la montée du national-socialisme, Mac Orlan se charge des problèmes sociaux et Dekobra explore le Milieu. Les reportages collectifs ${ }^{23}$, à angles de vue multiples, exploitent sous une autre forme la simultanéité du «panorama». C'est donc par un jeu - de déplacement, de surimpression des regards -, que le reportage réussit à dégager sa singularité, moins comme genre de l'objectivité que de la construction subjective, cherchant à endiguer la polyphonie des discours par le palimpseste des regards. 
13 Se renouvelant au gré des événements, l'actualité implique, d'autre part, une réalité en mouvement. Le reportage, censé capter l'histoire en train de se faire, tiendrait son authenticité de la peinture du transitoire, d'un réel non totalisable, mais en revanche, prédictible. La " puissance de "pronostication" ", selon une formule de Mac Orlan ${ }^{24}$, est au reporter ce que la réminiscence littéraire est à l'auteur du récit de voyage. Le sens du futur serait même un signe distinctif du genre. Contrairement, en effet, au récit de voyage qui fait du passé la mesure du présent ${ }^{25}$, le reportage privilégie ce que Cendrars nomme «l'actualité de demain ${ }^{26}$. Voir au futur pour mieux conjurer le passé : est-il, en effet, meilleur antidote à la redite que la prédiction? Face à la menace de l'intertexte, le reportage tente aussi de se constituer en "pré-texte », se maintenant par là-même au seuil de la littérarité.

La nouveauté, on le voit, est moins de l'ordre de l'innovation discursive que de l'invention scopique: si le récit de voyage s'entend comme regard sur l'autre, le reportage se veut, quant à lui, l'autre du regard. Il ne s'agit pas tant de se démarquer des modèles génériques (dont le reportage absorbe finalement les structures), que d'une subjectivité saturée de littérature: le reportage ne joue guère le jeu de la réécriture et de la variation stylistique, préférant voir autrement l'autre que dire autrement le même. C'est dans le déplacement du point de vue, dans la construction d'une subjectivité qui cherche à rendre compte de l'objet, que cette écriture de la " chose vue ", cherche une solution au "déjà dit ». Ce qui explique, pour une part, le caractère passablement conventionnel de ces récits, qui serait moins de l'ordre, esthétique, de la variation que de l'ordre, épistémologique, de la ressaisie.

Le reportage comme « littérature »?

Un pacte d'écriture qui ne manque cependant pas de placer le reportage dans une situation d'aporie à géométrie variable.

16 Tout d'abord, parce qu'à l'évidence, écrire contre la littérature, c'est à sa proie demeurer attaché, d'autre part parce que le flou poétique dont s'accommode le genre, offre aux auteurs une grande latitude de conception. Dans Sur la route Mandarine, Dorgelès entreprend un voyage à contre-lecture, soucieux de dégager le référent indochinois de son épaisseur référentielle, et plus particulièrement du modèle lotien, mais en se livrant... à un pastiche de Loti. Dans le chapitre plaisamment intitulé « Où je rencontre Pierre Loti en baie d'Along ", l'auteur des Croix de bois, met en scène le dilemme du reporter :

[...] l'âne de Buridan fut moins perplexe entre son seau et son picotin que l'écrivain, parfois, entre la réalité qui le conjure et la fiction qui le séduit...

Un lecteur impatienté. - Enfin, quoi, et cette congaïe ? Que devons-nous croire?

L'auteur. - Euh... C'est très ennuyeux ... Je voudrais bien vous être agréable et touner

à ma manière un conte qui fasse plaisir. Mais non, que voulez-vous, je ne peux pas...

Le lecteur. - Alors?

L'auteur. - Eh bien! tant pis. Elle avait les dents noires et elles sentait le poisson... ${ }^{27}$

Outre que le manifeste de l'anti-tradition littéraire s'achève en renouant avec Diderot, l'apologue ne semble guère bénéficier à la réalité qui, dans tous les sens du terme, laisse Dorgelès rêveur : " plus d'une fois, je me suis demandé si les plus beaux sites du monde ne seraient pas ceux dont on rêve toujours sans en approcher jamais ${ }^{28}$. Retour du refoulé imaginaire ? Reporter nostalgique du romancier? Dans Les Pirates de l'Avenue du Rhum (sous titré « reportage » lors de sa parution en volume chez Simon Kra en 1924), Mac Orlan évoque le piratage du cargo français le Mulhouse, à travers le souvenir des romans de Stevenson ou de Jack London et des Vies imaginaires de Marcel Schwob. 
L'actualité est là comme pour cautionner l'intertexte et pour lui faire suite. Dans ce qui n'est plus qu'une parodie de reportage, chiffres, dates et archives avèrent moins l'authenticité de l'enquête qu'ils ne font le jeu de l'imaginaire et au lieu de clarifier l'information, le récit s'emploie finalement à en épaissir le mystère.

De la réminiscence livresque à la parodie sur le vif, il n'y a qu'un pas que l'enquêteur franchit quand il entreprend de se rendre sur les lieux de la fiction pour y mettre le roman à l'épreuve du réel. C'est ainsi que Gaston Stiegler (pour Le Matin) et Henri Turot (pour Le Journal) se lancèrent, en 1901, sur les traces de Phileas Fogg pour un Tour du monde en 63 jours. Accueilli en gare d'Amiens par Jules Verne en personne, l'heureux recordman de la fiction, Stiegler, se voit attribuer par Gaston Leroux le titre, à y regarder de plus près, discutable, de " premier reporter du monde $»^{29}$.

La question de l'intertextualité qui se posait en termes d'incompatibilité avec le projet du témoignage, devient, non seulement une tentation littéraire, mais également une nécessité. Car qui entend témoigner d'un voir, ne saurait y parvenir, sauf à trahir la vocation informative de l'enquête, sans construire un savoir. Où finit alors le voir, où commence le savoir? Le reporter incorpore nécessairement des lectures au simple témoignage, quand bien même il ne s'agirait que du reportage lui-même qui s'interpose entre le vécu innocent et l'écriture informée. Dès lors qu'il se met en scène, le « regard naï » ne saurait être qu'une fiction. Lorsque Londres commence le récit de son enquête en disant : «J'arrivais. Je ne connaissais rien du bagne. J'étais bête " $^{30}$, ce n'est là qu'une feinte énonciative dans la tradition du roman de formation ou du conte philosophique. La route du bagne est parsemée de témoignages: Londres n'est par le premier journaliste à se rendre à Cayenne, et que dire de tous ceux qui, à sa suite, embarquent à bord du Biskra pour un voyage en terre écrite $?^{31}$ Difficile alors de transporter le lecteur au cœur du référent sans quelques détours par le déjà dit. Dans son avant-propos à Indochine SOS, Andrée Viollis rappelle qu'avant de partir, elle avait « été profondément émue par la belle et solide enquête de Louis Roubaud $»^{32}$ : comment, dans ce cas, séparer l'authentification d'une voir de la vérification d'un dire? Sans doute faut-il en conclure, avec Paul Nizan, que la connaissance immédiate est un leurre et que la réalité journalistique n'échappe pas à l'appréhension indirecte :

Il existe entre tout journal et les journaux dont la parution précède la sienne le même genre de rapport qu'entre un ouvrage de l'esprit et les ouvrages antérieurs de même ordre. Commandé pour l'essentiel par l'état externe des événements, le journal se définit cependant formellement par un rapport interne aux journaux antérieurement parus (...) tout journal prend une suite ${ }^{33}$.

Lorsque Béraud prétend que " pour juger les hommes, les nations, les événements [un Albert Londres, un Pierre Mille ou un Ludovic Naudeau] ne s'en remettaient qu'au témoignage de leurs yeux ${ }^{34}$, il s'agit nécessairement d'une image, la métaphore de «l'œil-objectif » passant sous silence la réalité d'un regard qui ne saurait s'exercer en l'absence de représentations préalables, nécessaires à l'intelligibilité même de la perception. Sans arrière-plan de connaissances, le reporter serait aveugle au fait. Le récit de Béraud, Ce que j'ai vu à Moscou, pourrait être sous-titré «ce que je sais de l'histoire russe et de la Révolution française ", tant l'auteur accumule, en historien, les parallèles, soulignant ainsi que pour voir il faut disposer d'une structure de "prévision » : "prévoir » ne signifie plus seulement «voir avant ", mais écrire d'après. Et parfois même plagier: c'est ainsi que dans Prisons de femmes (1933) Carco reprend textuellement un passage de Au bagne d'Albert Londres. Il y a loin du « regard neuf » au récit interchangeable. 
21 Le reportage tombe, dès lors, sous le coup d'un paradoxe majeur. Cette littérature en direct, de la plume trempée dans la vie, loin de refléter toujours, comme on serait en droit de l'attendre, la diversité du réel, apparaît fortement marquée par le stéréotype. Qu'il s'appelle Cendrars, Kessel ou Carco, le reporter qui enquête sur le Milieu n'est qu'un seul et même personnage : médiateur idéal entre le lecteur, dont il incarne le sens moral, et la canaille, avec laquelle il fraternise, il se présente comme un héros sans peurs et sans préjugés, un nouvel Orphée découvreur de mondes souterrains, remonté à la surface du journal, pour livrer au commun des mortels le secret de sa descente aux Enfers. Le modèle dantesque devient, parmi d'autres, l'un des archétypes les plus efficaces de la traversée des milieux occultes. Londres affiche la référence dans Dante n'avait rien $v u$, titre choisi pour l'édition en volume des articles sur Biribi, parus dans Le Petit Parisien; à chaque chapitre des Bas-fonds Kessel franchit un nouveau cercle de la pègre, à grand renfort de passeurs et de rites de passage, tout comme Londres explore le bagne selon une efficace gradation qui, du moins imaginable au plus inimaginable, construit l'information sur le modèle du récit initiatique. A ces mythes revisités, le reportage intègre des mythologies plus modernes comme la photographie ou le cinéma, qui intériorisent le modèle dont se réclame son écriture. Mac Orlan rend ainsi compte du piratage du Mulhouse, "à la manière d'un film joué par des personnages qui, pour avoir du talent, n'en risquent pas moins leur peau $»^{35}$ : de l'usage heuristique de la métaphore cinématographique pour dire «l'écran » à travers lequel le reporter voit, en fin de compte, le réel. Le reproche fut souvent adressé au genre, de pactiser trop étroitement avec la doxa. Citons pour exemple, parmi tant d'autres, cette savoureuse introduction d'Albert Londres aux asiles de femmes: "A côté des folles, les fous semblent raisonnables $»^{36}$. On n'en finirait pas d'énumérer les présupposés consensuels dont le reportage est émaillé.

«Les sirènes ne sont des monstres fabuleux que pour Homère qui, en définitive, n'était pas un reporter très sérieux. Ce sont tout simplement des lamentins $\|^{37}$ : Homère est un mauvais reporter, mais celui qui le dit, Albert Londres est une nouvelle Pénélope dans la mesure où le reportage refait ce qu'il défait, se désolidarisant de l'intertexte littéraire pour en reconstruire finalement le fantôme, sous la forme du stéréotype. En mettant l'accent sur la répétition, l'intertextualité, qui faisait la littérarité du récit de voyage, va contribuer à la sérialité du reportage, qu'il s'agisse du mode de publication en feuilleton ou de l'émergence de séries thématiques. Soucieux de se démarquer des modèles, le grand reportage finit par (re)devenir une " littérature » de " démarquage » (au sens où l'articlier " démarquait » jadis les faits divers en empruntant aux journaux concurrents), privilégiant une forme d'intertextualité restreinte au genre, et qui, de ce fait même, en constitue les signes de reconnaissance.

Roger Vailland considère que tout bon journaliste doit être attentif au «langage commun, auquel il convient d'attacher toujours la plus grande importance parce qu'il est le reflet, le produit et la conservation de la plus vaste expérience humaine ${ }^{38}$. En mobilisant le plus grand dénominateur culturel commun, le stéréotype gagne en force didactique, ce qu'il perd en nuance et en originalité littéraire, comme en fidélité au réel. Parce qu'il opère par réduction de l'inconnu au connu, de l'inhabituel au familier, le cliché répond à la vocation " grand public » du reportage, tenu de composer avec les savoirs et les représentations supposés du lecteur et/ou imposés par la ligne du journal. Alors même que le reporter se méfie de l'allusion littéraire comme d'un «coup de pistolet " dans le concert du référent, il accorde au stéréotype une valeur de 
transmission : le lieu commun, comme lieu de regards et de savoirs partagés, devient ainsi l'une des figures structurantes du genre.

L'intertextualité, qui s'oppose en son principe à la saisie immédiate du référent, est paradoxalement devenue, sous la forme extrême du stéréotype, un label d'origine et une marque de continuité du genre. Bien autant que le récit de voyage, le reportage met ainsi à nu la relation fondamentalement disjonctive qui unit le monde à sa représentation ${ }^{39}$. Pas moins que n'importe quel autre texte référentiel, il ne saurait échapper à ce destin d'altération de l'objet que menace tout discours sur lui, à plus forte raison lorsque, exploitant la veine du stéréotype, il réalise l'effacement de la singularité référentielle dans l'assomption du lieu commun.

L'intertexte à venir

Difficile de trouver la bonne distance entre mensonge réaliste et vérité intertextuelle. Plus encore lorsque le reporter est aussi un écrivain, et que c'est alors non seulement l'œuvre lue mais l'œuvre écrite ou à écrire qui entre en concurrence avec la «chose vue ». Les journaux annoncent le grand reportage des écrivains à grand bruit, déplaçant l'intérêt du lecteur, du contenu de l'enquête vers l'auteur du récit. La commande du journal à l'écrivain célèbre implique ainsi un double jeu énonciatif : l'auteur devra faire entendre le son de sa voix en même temps que la sonorité du réel. Dès lors, la réalité « vue par un tempérament » n'est plus séparable de l'actualité revue et corrigée par une œuvre. Pour la plupart des écrivains, le reportage va devenir un laboratoire de la création, et troquer son statut de témoignage de première main contre celui d'avant-texte ou d'intertexte à venir. Une démarche plus ou moins consciente de la part des auteurs qui ont diversement considéré la pratique du reportage : comme une école de la littérature (Audiberti, Leroux), une écriture de diversion (Soupault, Cendrars) ou au contraire d'accompagnement de l'œuvre (Mac Orlan). Prêtant à littérature - d'une manière qui confirme que la vision artiste ne lui était pas étrangère - le reportage préfigure, autant que le futur de l'Histoire, l'avenir d'une écriture.

Simenon, qui ne voyait dans le reportage qu' « une façon de financer [...] ses curiosités » ${ }^{40}$, découvre sur le tard, que ses écrits de journalisme, tout juste considérés comme une d'exutoire de l'œuvre, « [contenaient] en germe tous les romans [qu'il a] conçus par la suite $»^{41}$. Plus délibérée est la démarche de Malraux, lorsqu'il truffe L'Espoir d'extraits de reportages contemporains ou d'Aragon lorsqu'il insère dans les romans du Monde réel des collages d'articles parus dans L'Humanité. Kessel le reporter «fait provision de personnages, de descriptions, de scènes et d'informations $\gg^{42}$ pour le romancier, tandis que Cendrars, remanie ses enquêtes sur Hollywood ou le Brésil, en s'efforçant de les réaffilier à l'œuvre, notamment par un système d'allusions rétrospectives aux romans antérieurs. Toutefois, à la différence de Cendrars citant Mathurin Régnier en plein Panorama de la pègre, Kessel demeure un érudit discret dans l'enquête, en écrivain qui joue des deux plumes sans les confondre. De même, faisant d'un seul voyage deux livres, Dorgelès double le récit de reportage (Sur la route mandarin, 1925) du roman du voyage (Partir, 1926). Roger Vailland au contraire, romance le reportage (La Visirova ou des Folies-Bergère jusqu'au trône $e^{43}$ ) dont il fait un ban d'essai pour romancier en mal d'invention. Autant de variations sur une même esthétique de la reprise, qui tendent à faire du reportage un genre de l'emprunteur emprunté. Quand il n'est pas d'emblée un faux jumeau du journalisme, de l'ordre du pastiche, le reportage devient matière première du roman et, ainsi voué au recyclage, préfigure la fiction dans le temps même où il rend compte du réel. Devenu métaphore d'un certain regard sur le monde, mais 
aussi citation - destin inattendu d'un genre héritier de la "tranche de vie » - le reportage se disperse alors dans la littérature, au risque de s'y perdre.

Témoin d'un malaise dans la création, que ce soit celui de l'engagement ou de la représentation, le reportage semble avoir occupé le temps d'une crise de la littérature, qui se serait résolue au bénéfice de la littérature, si l'on en croit Malraux livrant dès 1935 ce constat dans sa préface à Indochine SOS d'Andrée Viollis :

Je pense qu'il est bien peu de romanciers de notre temps qui n'aient rôdé autour des reportages réunis en volumes, qui n'aient senti que se préparait là une forme nouvelle de roman, et qui n'aient assez vite abandonné leur espoir ${ }^{44}$.

Le même Malraux, impatienté par la question mainte fois soulevée de la dette de reportage dont sont redevables plusieurs de ses romans, répondait à Denis Marion par une boutade: "Qui "aujourd'hui" confondrait [mes] livres avec les cadavres des reportages de l'époque ? $\aleph^{45}$. Une maigre obole à une forme de récit qui a pris une part active à cette "ère du soupçon" dont le roman sortira renouvelé: littérature des choses (Pierre Hamp, Jean-Richard Bloch), roman de la conscience engagée (Nizan, Malraux) ou même nouveau roman. Un destin qui rejoindrait alors, pour une large part, celui des productions populaires, intertextes de prédilection d'une littérature au second degré, parfois oublieuse de ses emprunts.

Né à l'ère de l'utopie du direct le reportage, qui se définissait comme « littérature » du premier degré, n'a en fait cessé de se construire en absorbant et en remaniant d'autres genres, le récit de voyage et le roman mais également la biographie et l'autobiographie dont il resterait également à démontrer la contribution. Ce genre pillard n'a pas manqué, à son tour, d'être pillé, par le roman mais aussi par l'essai ou la chronique et même la poésie, qui l'ont intégré à leur esthétique comme "une composante de l'écriture $~^{46}$. Tout à la fois rapsodique et disséminé, le reportage, s'est construit au péril de ses frontières comme une forme d'écriture doublement passagère : qui d'une part a composé avec les genres, d'autre part ne s'est pas durablement imposée. Ce rôle de plaque-tournante des genres, auquel semble, à distance, se résumer pour une part le reportage, en fait finalement un lieu de haute intertextualité, mettant en évidence les opérations de passage, d'échange ou de transfert à l'œuvre dans l'invention, toujours à réinventer, de la prose du monde.

\section{NOTES}

1. Paul Morand à propos des Conquérants de Malraux, cité par Eliane Tonnet-Lacroix, La Littérature française de l'entre-deux-guerres (1919-1939), Paris, Nathan, 1993, p. 28.

2. Jacques Rivière, «La crise du concept de littérature ", La Nouvelle Revue française, $\mathrm{n}^{\circ} 125,1$ er février 1924.

3. Pierre Mac Orlan, «Les compagnons de l'aventure : correspondants de guerre et grands reporters » (1928), repris dans Le Mystère de la malle $n^{\circ} 1$ et autres reportages, Paris, 10/18, coll. « Grands reporters », 1984, p. 234. 
4. Henri Béraud, préface au Flâneur salarié, Paris, Les Editions de France, 1927, p. XIII et XV.

5. L'expression est de Paul Morand dans Chroniques de l'homme maigre, Paris, Grasset, 1941, p. 120.

6. Albert Londres, dédicace de Au Bagne (Le Petit Parisien, août-septembre 1923), rééd. Paris, Le Serpent à plumes, coll. « Motifs », 1998.

7. François Hourmant, Au pays de l'avenir radieux, Voyages des intellectuels français en URSS, à Cuba et en Chine populaire, Paris, Aubier, coll. « Historique », 2000.

8. Henri Béraud, Le Flâneur salarié, op. cit., p. XII.

9. Paul Morand, Chroniques de l'homme maigre, op. cit., p. 120.

10. Cité par Michel Leymarié, « Les frères Tharaud et le journalisme ", L'Ecrivain journaliste, Paris, Klincksieck, coll. "Littératures contemporaines ", n 6, 1998, p. 163. 11. Roland Dorgelès, La caravane sans chameaux, Paris, Albin Michel, 1928, p. 20.

12. Voir Dominique Maingueneau, Le Contexte de l'œuvre littéraire, Enonciation, écrivain, société, Paris, Dunod, 1993, chap. 6.

13. L'expression revient souvent : «En définitive, il s'agit bien moins de renseigner le public que de le tenir en haleine » (Jean Rodes, « Le roman reportage », Le Journal littéraire, n 9, 21 juin 1924 ; « Le bon reporter doit [...] tenir le lecteur en haleine, comme tout ce qu'il fait et tout ce qu'il entend le tient lui-même dans un état de curiosité un peu fiévreux » (Jérôme et Jean Tharaud, préface à Grands reportages, Paris, Corrêa, coll. « Les grandes professions françaises », 1946, p. 16).

14. Joseph Kessel, «J'ai toujours voulu rester libre », Magazine littéraire, $n^{\circ} 32$, Kessel Journalisme et Littérature, septembre 1969, p. 10.

15. Joseph Kessel, Bas-fonds, Editions des Portiques, 1932, p. 27.

16. André Salmon, Souvenirs sans fin, Deuxième époque (1908-1920), Paris, Gallimard, 1956, p. 316.

17. Jean Rodes, op. cit.

18. Véronique Magri, Le Discours sur l'autre, A travers quatre récits de voyage en Orient, Paris, Honoré Champion, 1995, p. 134.

19. Ibid., p. 128-129 et 204-205.

20. Voir Francis Carco, Prisons de femmes, Paris, Les Editions de France, 1933 et Les Hommes en cage, Paris, Albin Michel, 1936.

21. Voir l'enquête de Georges Simenon, « En marge de l'affaire Stavisky », Paris-Soir, Janvier-février 1934.

22. Blaise Cendrars, Panorama de la pègre (1935), CEuvres complètes, t. 4, Paris, Denoël, 1960, p. 288. Sur l'esthétique du « panorama », voir Renaud Ferreira de Oliveira, « Kessel, Cendrars : deux versions de la pègre », Littérature et reportage, actes du colloque de Limoges (26-28 avril 2000) réunis par Myriam Boucharenc et Joëlle Deluche, Presses Universitaires de Limoges (à paraître).

23. La formule sera reprise à la veille du scrutin de 1936. Cinq reporters parcourent pour Paris-Soir « la France qui vote » (29 articles parus durant le mois d'avril 1936).

24. Pierre Mac Orlan, « Albert Londres et les grands reporters » (1954), repris dans Les Compagnons de l'aventure, choix et préface de Francis Lacassin, Paris, Editions du Rocher, 1997, p. 216.

25. Véronique Magri, op. cit., p. 321.

26. «L'actualité de demain » est le titre d'une nouvelle d'Histoires vraies (1937).

27. Roland Dorgelès, Sur la route mandarine, Paris, Albin Michel, 1925, p. 77.

28. Ibid., p. 8. 
29. Alfu, Gaston Leroux, Parcours d'une œuvre, Paris, Encrage, coll. « Références », 1996, p. 35 .

30. Albert Londres, Au bagne, op. cit., p. 25.

31. Citons à titre indicatif : Georges Le Fèvre, Bagnards et chercheurs d'or (1925), J.-

F. Louis Merlet, Au bout du monde, Drames et misères du bagne (1928), Marius Larique, Les Hommes punis (1931), Paul Darlix, Avec les « Durs» (1932).

32. Il s'agit de l'enquête parue aux éditions Valois, sous le titre Viet-Nam. Voir Andrée Viollis, Indochine SOS, Paris, Gallimard, 1935, p. XIII.

33. Paul Nizan, Chronique de septembre, Paris, Gallimard, 1978, p. 10.

34. Henri Béraud, Les Derniers Beaux Jours, Paris, Plon, 1953, p. 55.

35. Pierre Mac Orlan, Les Pirates de l'Avenue du Rhum (1924), repris dans Le Mystère de la malle $n^{\circ} 1$ et autres reportages, op. cit., p. 88.

36. Albert Londres, Chez les fous (1925), rééd. Paris, Le Serpent à plumes, 1999, p. 38.

37. Albert Londres, Au bagne, op. cit., p. 206.

38. Roger Vailland, Chronique d'Hiroshima à Goldfinger (1945-1965), Paris, Messidor, 1984, p. 16.

39. Voir le bel essai de Christine Montalbetti, Le Voyage, le monde et la bibliothèque, Paris, Presses Universitaires de France, 1997, p. 5.

40. Cité par Francis Lacassin, Simenon, L'homme, l'univers, la création, Paris, Editions Complexe, 1993, p. 68.

41. Georges Simenon, « Mes dictées », Mémoires, t. 2, Presses de la Cité, 1993, p. 1218.

42. Alain Tassel, La Création romanesque dans l'œuvre de Joseph Kessel, Paris, L'Harmattan, 1997, p. 393.

43. L'ambiguïté générique est à son comble lorsque Paris-Soir (juillet-août 1933) publie « le plus extraordinaire roman vécu en 1933, La Visirova ou des Folies Bergères jusqu'au trône, grand reportage par Roger Vailland » (repris aux Editions Messidor en 1986).

44. André Malraux, préface à Andrée Viollis, Indochine SOS, op. cit., p. VII.

45. André Malraux, cité par Christiane Moatti, «L'Histoire dans un roman d'André Malraux : reportage ou mythe? ", Irruption de l'histoire dans la littérature française de l'entre-deux-guerres, textes réunis par René Garguilo et Abraham Ablamowicz, Universités de Silésie et de la Sorbonne nouvelle, 1986, p. 123.

46. Catherine Milkovitch-Rioux, " "J'écris dans la tempête” : les échos de la guerre d'Algérie ", Littérature et reportage, op. cit.

\section{RÉSUMÉS}

Le reportage qui s'impose dès la Belle Epoque comme le genre phare de la grande presse d'information, est en passe de devenir durant l'entre-deux-guerres un nouveau genre littéraire : la tentation de l'enquête touche alors un nombre considérable d'écrivains et non des moindres Colette, Cendrars, Dorgelès, Carco, Soupault, Vailland... - pour n'en citer que quelques-uns. L'ambiguïté du genre tient notamment à sa double postulation, l'une vers la littéralité (la soumission à la «chose vue »), l'autre vers la littérarité : la chose "écrite» mais aussi «lue». Partant, le reportage entretient un rapport hésitant et duplice avec le jeu intertextuel qui, 
variablement assumé, semble remplir différentes fonctions parmi lesquelles une fonction polémique (de démarcation par rapport aux genres mitoyens du roman d'aventures et du récit de voyage), une fonction poétique s'exerçant dans le paradigme restreint d'un genre à la recherche de ses rituels d'écriture propres, une fonction génétique enfin, dans la mesure où l'œuvre de l'écrivain bien souvent s'amorce, se poursuit ou se réfléchit dans l'écriture du reportage.

\section{AUTEUR}

MYRIAM BOUCHARENC

Université de Limoges 